J. Korean Math. Soc. 52 (2015), No. 3, pp. 649-661

http://dx.doi.org/10.4134/JKMS.2015.52.3.649

\title{
INSERTION-OF-FACTORS-PROPERTY WITH FACTORS MAXIMAL IDEALS
}

\author{
Hai-Lan Jin, Da Woon Jung, Yang Lee, Sung Ju Ryu, Hyo Jin Sung, \\ AND SANG Jo YUN
}

\begin{abstract}
Insertion-of-factors-property, which was introduced by Bell, has a role in the study of various sorts of zero-divisors in noncommutative rings. We in this note consider this property in the case that factors are restricted to maximal ideals. A ring is called $I M I P$ when it satisfies such property. It is shown that the Dorroh extension of $A$ by $K$ is an IMIP ring if and only if $A$ is an IFP ring without identity, where $A$ is a nil algebra over a field $K$. The structure of an IMIP ring is studied in relation to various kinds of rings which have roles in noncommutative ring theory.
\end{abstract}

\section{Introduction}

Throughout this note every ring is an associative ring with identity unless otherwise stated. Given a ring $R, N_{0}(R), N_{*}(R), N^{*}(R), N(R), \mathcal{B} R(R)$, and $J(R)$ denote the Wedderburn radical (the sum of all nilpotent ideals), the prime radical, the upper nilradical (i.e., the sum of all nil ideals), the set of all nilpotent elements, the Brown-McCoy radical (i.e., the intersection of all maximal ideals), and the Jacobson radical of $R$, respectively. Note $N_{0}(R) \subseteq$ $N_{*}(R) \subseteq N^{*}(R) \subseteq N(R)$ and $N^{*}(R) \subseteq J(R) \subseteq \mathcal{B} R(R)$. The $n$ by $n$ full (resp., upper triangular) matrix ring over $R$ is denoted by $\operatorname{Mat}_{n}(R)$ (resp., $U_{n}(R)$ ), and denote by $E_{i j}$ the matrix with $(i, j)$-entry 1 and elsewhere zero. $\mathbb{Z}$ denotes the ring of integers, and $\mathbb{Z}_{n}$ denotes the ring of integers modulo $n$. For a ring $R, R[x]$ (resp., $R[[x]]$ ) denotes the polynomial (resp., power series) ring with an indeterminate $x$ over $R$. For $f(x) \in R[x]$, let $C_{f(x)}$ denote the set of all coefficients of $f(x)$.

Insertion-of-factors-property has done important roles in noncommutative ring theory and module theory. Due to Bell [3], a ring $R$ (possibly without identity) is called to satisfy the insertion-of-factors-property (simply, an IFP ring) if $a b=0$ implies $a R b=0$ for $a, b \in R$. Narbonne [15] and Shin [16] used the terms semicommutative and SI for the IFP, respectively. It is easily

\footnotetext{
Received October 17, 2014.

2010 Mathematics Subject Classification. 16D25.

Key words and phrases. IMIP ring, maximal ideal, IFP ring, Dorroh extension, idempo-
} tent. 
checked that $N_{*}(R)=N(R)$ when $R$ is an IFP ring. The class of IFP rings is closed under subrings obviously. A ring $R$ (possibly without identity) is called reduced if $N(R)=0$. The class of IFP rings contains both commutative rings and reduced rings. But there exist many non-reduced commutative rings (e.g., $\mathbb{Z}_{n^{l}}$ for $n, l \geq 2$ ), and many noncommutative reduced rings (e.g., direct products of noncommutative domains). A ring is usually called Abelian if each idempotent is central. It is easily checked that IFP rings are Abelian.

The concept of IFP does not pass to polynomial rings by [9, Example 2]. But we have the following fact related to (maximal) ideals of the ground ring $R$ when $R[x]$ is IFP.

Remark 1.1. Let $R$ be a ring. The following conditions are equivalent:

(1) $R[x]$ is IFP;

(2) $x^{n} R[x]$ is an IFP ring for all $n \geq 1$;

(3) $M+x^{n} R[x]$ is an IFP ring for all (maximal) ideals $M$ of $R$ and $n \geq 1$.

Proof. $(1) \Rightarrow(3)$ and $(3) \Rightarrow(2)$ are obvious, so it suffices to show $(2) \Rightarrow(1)$. Assume that the condition (2) holds. Let $f(x) g(x)=0$ for $f(x), g(x) \in R[x]$. Then $\left(x^{n} f(x)\right)\left(x^{n} g(x)\right)=0$. Since $x^{n} R[x]$ is IFP, $\left(x^{n} f(x)\right)\left(x^{n} h(x)\right)\left(x^{n} g(x)\right)=$ 0 for all $h(x) \in R[x]$. This yields $f(x) h(x) g(x)=0$, proving that $R[x]$ is IFP.

In Remark 1.1, $a b=0$ for $a, b \in R$ implies $a M b=0$ for any ideal $M$ of $R$. We now introduce the following definition.

Definition 1.2. A ring $R$ is said to satisfy the insertion-of-maximal-idealproperty (simply, an IMIP ring) if $a M b=0$ for some maximal ideal $M$ of $R$ whenever $a b=0$ for all $a, b \in R$.

Every simple ring is clearly IMIP, we note that there exist many non-simple IFP rings (e.g., $\mathbb{Z}$ ). IFP rings are clearly IMIP, the converse is not true by the existence of non-Abelian simple rings (e.q., $\operatorname{Mat}_{2}\left(\mathbb{Z}_{2}\right)$ ).

Proposition 1.3. (1) If $R$ is an IMIP ring, then $N_{0}(R)=N_{*}(R)=N^{*}(R)$.

(2) If $R$ is an IMIP ring with $J(R)$ nil, then $N_{0}(R)=N_{*}(R)=N^{*}(R)=$ $J(R)$.

(3) If $R$ is an IMIP ring in which every prime ideal is maximal, then $N_{0}(R)=N_{*}(R)=N^{*}(R)=J(R)=\mathcal{B} R(R)$.

(4) If $R$ is an IFP ring, then $N_{0}(R)=N_{*}(R)=N^{*}(R)=N(R)$.

(5) Let $R$ be a ring such that $R$ has distinct maximal ideals $M_{1}, M_{2}$ satisfying $M_{1} \cap M_{2}=0$. Then each $M_{i}$ is an IFP ring without identity if and only if $R$ is IFP.

Proof. (1) Let $R$ be an IMIP ring and $a \in N^{*}(R)$. Then $a^{n}=0$ for some $n \geq 1$. Since $R$ is IMIP and every maximal ideal of $R$ contains $N^{*}(R)$, we have $a(R a R) a^{n-1}=0$ and so for any $b_{1} \in R a R$, we have $a b_{1} a(R a R) a^{n-2}=0$. Continuing in this manner, we finally get

$$
a b_{1} a b_{2} a \cdots a b_{n-1} a=0
$$


where $b_{i} \in R a R$ for $i=1,2, \ldots, n-1$. This yields $(R a R)^{2 n-1}=0$, entailing $a \in N_{0}(R)$.

(2) and (3) come from (1).

(4) is obvious, since any IFP ring $R$ is $\operatorname{IMIP}$ with $N_{*}(R)=N(R)$.

(5) It suffices to show the necessity. Let $a b=0$ for $a, b \in R$. Since $M_{1}+M_{2}=$ $R, a=a_{1}+a_{2}$ and $b=b_{1}+b_{2}$ for some $a_{1}, b_{1} \in M_{1}$ and $a_{2}, b_{2} \in M_{2}$. From $M_{1} \cap M_{2}=0$, we get $0=a b=\left(a_{1}+a_{2}\right)\left(b_{1}+b_{2}\right)=a_{1} b_{1}+a_{1} b_{2}+a_{2} b_{1}+a_{2} b_{2}=$ $a_{1} b_{1}+a_{2} b_{2}$. This yields $a_{1} b_{1}=-a_{2} b_{2} \in M_{1} \cap M_{2}=0$, entailing $a_{1} b_{1}=0$ and $a_{2} b_{2}=0$. But since each $M_{i}$ is IFP, we have $a_{1} M_{1} b_{1}=0$ and $a_{2} M_{2} b_{2}=0$. Note that both $a_{1} M_{2} b_{1}$ and $a_{2} M_{1} b_{2}$ are contained in $M_{1} \cap M_{2}$, so each of them must be zero. Consequently we have

$$
a_{1} R b_{1}=a_{1}\left(M_{1}+M_{2}\right) b_{1}=0 \text { and } a_{2} R b_{2}=a_{2}\left(M_{1}+M_{2}\right) b_{2}=0 .
$$

So, for all $r \in R$, we obtain

$a r b=\left(a_{1}+a_{2}\right) r\left(b_{1}+b_{2}\right)=a_{1} r b_{1}+a_{1} r b_{2}+a_{2} r b_{1}+a_{2} r b_{2}=a_{1} r b_{1}+a_{2} r b_{2}=0$, noting $a_{1} r b_{2}, a_{2} r b_{1} \in M_{1} \cap M_{2}$. Thus $R$ is IFP.

In the following one can see a ring which satisfies (2) and (3) in Proposition 1.3. Let $A$ be an algebra (with or without identity) over a commutative ring $S$. Due to Dorroh [5], the Dorroh extension of $A$ by $S$ is the Abelian group $D=A \oplus S$ with multiplication given by

$$
\left(r_{1}, s_{1}\right)\left(r_{2}, s_{2}\right)=\left(r_{1} r_{2}+s_{1} r_{2}+s_{2} r_{1}, s_{1} s_{2}\right),
$$

where $r_{i} \in A$ and $s_{i} \in S$.

Example 1.4. Let $K$ be a field and $A$ be a nonzero algebra over $K$ such that $A^{2}=0$ (e.g., $\left.A=\left(\begin{array}{cc}0 & K \\ 0 & 0\end{array}\right) \subset U_{2}(K)\right)$. Set $R$ be the Dorroh extension of $A$ by $K$. Let $(s, t) \in R$ with $t \neq 0$. Then $(s, t)$ is a unit in $R$ with $(s, t)^{-1}=\left(-t^{-2} s, t^{-1}\right)$. This implies that $A \oplus\{0\}$ is the unique maximal ideal of $R, M$ say. Therefore $R$ is a commutative ring with $N_{0}(R)=N_{*}(R)=N^{*}(R)=J(R)=\mathcal{B} R(R)=M$.

In the following we observe a connection between the IMIP and the IFP.

Proposition 1.5. Let $A$ be a nil algebra over a field $K$, and $R$ be the Dorroh extension of $A$ by $K$. Then $R$ is IMIP if and only if $A$ is an IFP ring without identity.

Proof. We first claim that every $(x, y) \in R$ is a unit if $y \neq 0$. Say $x^{n}=0$ for some integer $n \geq 2$. Then $\left(x y^{-1}\right)^{n}=0$, and this yields

$$
\begin{aligned}
& \left(x y^{-1}, 1\right)\left((0,1)-\left(x y^{-1}, 0\right)+\cdots+(-1)^{n-1}\left(x y^{-1}, 0\right)^{n-1}\right) \\
= & \left((0,1)+\left(x y^{-1}, 0\right)\right)\left((0,1)-\left(x y^{-1}, 0\right)+\cdots+(-1)^{n-1}\left(x y^{-1}, 0\right)^{n-1}\right) \\
= & \left((0,1)+\left(x y^{-1}, 0\right)\right)\left((0,1)-\left(x y^{-1}, 0\right)+\cdots+(-1)^{n-1}\left(\left(x y^{-1}\right)^{n-1}, 0\right)\right) \\
= & (0,1) .
\end{aligned}
$$


Similarly $\left((0,1)-\left(x y^{-1}, 0\right)+\cdots+(-1)^{n-1}\left(x y^{-1}, 0\right)^{n-1}\right)\left((0,1)+\left(x y^{-1}, 0\right)=\right.$ $(0,1)$. Thus we have

$$
(x, y)^{-1}=\left(0, y^{-1}\right)\left((0,1)-\left(x y^{-1}, 0\right)+\cdots+(-1)^{n-1}\left(x y^{-1}, 0\right)^{n-1}\right),
$$

noting $(x, y)\left(0, y^{-1}\right)=\left(x y^{-1}, 1\right)$. The above claim leads that $A \oplus\{0\}$ is the unique maximal ideal of $R, M$ say.

Suppose that $a b=0$ for $a, b \in A$. Then $(a, 0)(b, 0)=0$. If $R$ is IMIP, then $0=(a, 0) M(b, 0)=(a A b, 0)$, recalling that $M$ is the unique maximal ideal of $R$. So we get $a A b=0$, proving that $A$ is IFP.

Conversely, let $A$ be an IFP ring and suppose that $(a, c)(b, d)=0$ for $(a, c),(b, d) \in R$. Then $c=d=0$ by the claim above; hence $a b=0$. Since $A$ is IFP, we have $a A b=0$. This yields $(a, c) M(b, d)=(a A b, 0)=0$, proving that $R$ is IMIP.

One may ask whether the IMIP may pass to Dorroh extensions, considering Proposition 1.5. But the following argument answers negatively. Consider the simple nil algebra $\bar{A}$ over a field $K$ constructed by Smoktunowicz [17, Theorem 6.6]. Then $\bar{A}$ is an IMIP ring without identity. Note that $\bar{A}$ has a nilpotent element $a$ with $a^{2}=0$ and $a b a \neq 0$ for $b \in \bar{A}$. Here assume that the Dorroh extension $R$ of $\bar{A}$ by $K$ is IMIP. Then $(a \bar{A} a, 0)=(a, 0)(\bar{A} \oplus 0)(a, 0)=0$, a contradiction. Thus $R$ cannot be IMIP.

In the following we see a relation between simple and IMIP, via matrix rings.

Theorem 1.6. A ring $R$ is simple if and only if $\operatorname{Mat}_{n}(R)$ is an IMIP ring for all $n \geq 2$.

Proof. If $R$ is a simple ring, then $\operatorname{Mat}_{n}(R)$ is also simple (hence IMIP) for all $n \geq 2$.

Conversely, let $M=\operatorname{Mat}_{n}(R)$ be an IMIP ring for $n \geq 2$ and assume on the contrary that $R$ is not simple. Let $j \neq s$ for $j, s \in\{1,2, \ldots, n\}$. Then $E_{i j} E_{s t}=0$ for all $i, t \in\{1,2, \ldots, n\}$. Since $M$ is IMIP and non-simple, there exists a nonzero maximal ideal $I$ of $M$ such that $E_{i j} I E_{s t}=0$. This yields $M E_{i j} M I M E_{s t} M=0$. Let $0 \neq\left(\alpha_{x y}\right) \in I$ such that $\alpha_{u v} \neq 0$. Then

$$
0 \neq \alpha_{u v} E_{11}=E_{1 i} E_{i j} E_{j 1} E_{1 u}\left(\alpha_{x y}\right) E_{v 1} E_{1 s} E_{s t} E_{t 1} \in M E_{i j} M I M E_{s t} M=0,
$$

a contradiction. Thus such a nonzero maximal ideal $I$ cannot exist in $M$, and so $R$ is simple.

By help of Theorem 1.6, we have the following remark.

Remark 1.7. Let $M=\operatorname{Mat}_{n}(R)$ over a simple ring $R$. Then:

(1) $M$ is IMIP;

(2) $0=N_{*}(M) \subsetneq N(M)$, comparing with the property that $N_{*}(A)=N(A)$ for any IFP $\operatorname{ring} A$;

(3) $M$ is semiprime but not reduced, comparing with the fact that a ring is reduced if and only if it semiprime IFP. 
The following example shows that $U_{n}(R)$ cannot be IMIP for any $\operatorname{ring} R$ and $n \geq 2$.

Example 1.8. Consider a $\operatorname{ring} R=U_{n}(R)(n \geq 2)$ over any $\operatorname{ring} R$. Note that any maximal ideal of $U_{n}(R)$ is one of the form

$$
\left(\begin{array}{cccc}
M & R & \cdots & R \\
0 & R & \cdots & R \\
\vdots & \vdots & \ddots & \vdots \\
0 & 0 & \cdots & R
\end{array}\right),\left(\begin{array}{cccc}
R & R & \cdots & R \\
0 & M & \cdots & R \\
\vdots & \vdots & \ddots & \vdots \\
0 & 0 & \cdots & R
\end{array}\right), \ldots, \text { or }\left(\begin{array}{cccc}
R & R & \cdots & R \\
0 & R & \cdots & R \\
\vdots & \vdots & \ddots & \vdots \\
0 & 0 & \cdots & M
\end{array}\right)
$$

where $M$ denotes any maximal ideal of $R$. For $E_{11}, E_{22} \in U_{n}(R)$, we have $E_{11} E_{22}=0$. But any maximal ideal of $U_{n}(R)$ contains $E_{12}$ and $E_{11} E_{12} E_{22}=$ $E_{12} \neq 0$. This shows that $U_{n}(R)$ is not IMIP.

Example 1.8 says that the class of IMIP rings is not closed under subrings, noting that $\operatorname{Mat}_{n}(R)$ over any simple $\operatorname{ring} R$ for $n \geq 2$ is an IMIP ring by Theorem 1.6.

Proposition 1.9. If $R$ is an IMIP ring, then eRe is IMIP for all $0 \neq e^{2}=$ $e \in R$.

Proof. Let $R$ be an IMIP ring and $0 \neq e^{2}=e \in R$. Suppose $a b=0$ for $a, b \in e R e$. Then $a e=a$ and $e b=b$. Since $R$ is IMIP, $a M b=0$ for some maximal ideal $M$ of $R$. Note $a M b=a e M e b$. If $e M e=e R e$, then $a N b=0$ for all maximal ideals $N$ of $e R e$. So assume $e M e \subsetneq e R e$. Since $e M e \subseteq$ $(e R e) M(e R e)=e \operatorname{Re}(R M R) e R e$ and $e R e(R M R) e R e \subseteq e M e$, we have

$$
e M e=e \operatorname{Re}(R M R) e R e .
$$

We next show that $e M e$ is a maximal ideal of $e R e$. One can find the proof by help of [8, Theorem 3], but we here write another one. Assume that $e M e \subsetneq N_{1}$ for some ideal $N_{1}$ of $e$ Re. Then

$$
N_{1}=e R e N_{1} e R e=e R N_{1} R e,
$$

since $e N_{1} e=N_{1}$. If $R N_{1} R=R M R$, then

$$
e M e=e \operatorname{Re}(R M R) e \operatorname{Re}=e \operatorname{Re}\left(R N_{1} R\right) e R e=e R N_{1} R e=e N_{1} e=N_{1},
$$

a contradiction. This forces $M=R M R \subsetneq R N_{1} R=R$ since $M$ is maximal in $R$. It then follows that

$$
N_{1}=e R e N_{1} e R e=e R N_{1} R e=e R e .
$$

Thus $e M e$ is a maximal ideal of $e R e$. Moreover $0=a M b=a(e M e) b$ since $a=a e$ and $b=e b$, proving that $e$ Re is IMIP.

The converse of Proposition 1.9 does not hold in general. Recall the nonIMIP ring $R=U_{2}(\mathbb{Z})$ in Example 1.8. For an idempotent $e=E_{11} \in R$, $e R e \cong \mathbb{Z}$ is IMIP clearly. 
Following [6], a ring $R$ is called (von Neumann) regular if for each $a \in R$ there exists $b \in R$ such that $a=a b a$. For a regular ring $R, R$ is reduced if and only if $R$ is IFP if and only if $R$ is Abelian by help of [6, Theorem 3.2].

Considering Proposition 1.3(1),(2), one may conjecture that regular IMIP rings are reduced. However we note that $\operatorname{Mat}_{2}\left(\mathbb{Z}_{2}\right)$ is regular IMIP, which not reduced.

A ring $R$ is called directly finite if $a b=1$ implies $b a=1$ for $a, b \in R$. Clearly Abelian rings (e.g., IFP rings) are directly finite. So one may conjecture that IMIP rings are directly finite. However there exists a simple ring that is not directly finite by $[7$, Theorem 1.3$]$.

\section{Properties of IMIP rings related to ring extensions}

In this section we examine the IMIP property of several ring extensions.

Given a ring $R$ and an $(R, R)$-bimodule $M$, the trivial extension of $R$ by $M$ is the ring $T(R, M)=R \oplus M$ with the usual addition and the following multiplication: $\left(r_{1}, m_{1}\right)\left(r_{2}, m_{2}\right)=\left(r_{1} r_{2}, r_{1} m_{2}+m_{1} r_{2}\right)$. This is isomorphic to the ring of all matrices $\left(\begin{array}{cc}r & m \\ 0 & r\end{array}\right)$, where $r \in R$ and $m \in M$ and the usual matrix operations are used.

Notice that if $R$ is a reduced ring, then $T(R, R)$ is IMIP by help of [14, Proposition 1.6]. But the following example illuminates that the trivial extension of an IMIP ring need not be so.

Example 2.1. Let $R=\operatorname{Mat}_{2}\left(\mathbb{Z}_{2}\right)$. Then $R$ is IMIP by Theorem 1.6. Consider the trivial extension $T(R, R)$, then the only maximal ideal of $T(R, R)$ is

$$
M=\left(\begin{array}{cc}
0 & R \\
0 & 0
\end{array}\right),
$$

since $R$ is simple. Take

$$
A=\left(\begin{array}{ll}
a & 0 \\
0 & a
\end{array}\right) \in T(R, R),
$$

for $a=\left(\begin{array}{ll}1 & 1 \\ 1 & 1\end{array}\right) \in R$. Then $A^{2}=0$, since $a^{2}=0$. For

we have

$$
\alpha=\left(\begin{array}{cc}
0 & m_{1} \\
0 & 0
\end{array}\right) \in M \text { where } m_{1}=\left(\begin{array}{ll}
0 & 1 \\
0 & 0
\end{array}\right) \in R,
$$

$$
\left.A \alpha A=\left(\begin{array}{cc}
0 & a \alpha a \\
0 & 0
\end{array}\right)=\left(\begin{array}{ll}
0 & a \\
0 & 0
\end{array}\right)=\left(\begin{array}{ll}
0 & 0 \\
0 & 0 \\
0 & 0 \\
0 & 0
\end{array}\right) \quad\left(\begin{array}{ll}
1 & 1 \\
1 & 1
\end{array}\right)\right) \neq 0
$$

entailing that $A M A \neq 0$ and therefore $T(R, R)$ is not IMIP.

For a reduced ring $R$ and $f(x), g(x) \in R[x]$, Armendariz [2, Lemma 1] proved that

$$
a b=0 \text { for all } a \in C_{f(x)}, b \in C_{g(x)} \text { whenever } f(x) g(x)=0 .
$$


Chhawchharia and Rege [4] called a ring Armendariz if it satisfies this property. So reduced rings are clearly Armendariz. This fact will be used freely in this note. Armendariz rings are Abelian by the proof of [1, Theorem 6] (or [13, Lemma 7]). The concepts of Armendariz and IFP are independent of each other by [4, Example 3.2] and [9, Example 14].

Let $R$ be a ring and $n \geq 2$, define

$$
D_{n}(R)=\left\{\left(\begin{array}{ccccc}
a & a_{12} & a_{13} & \cdots & a_{1 n} \\
0 & a & a_{23} & \cdots & a_{2 n} \\
0 & 0 & a & \cdots & a_{3 n} \\
\vdots & \vdots & \vdots & \ddots & \vdots \\
0 & 0 & 0 & \cdots & a
\end{array}\right) \in U_{n}(R) \mid a, a_{i j} \in R\right\} .
$$

Note that $D_{2}(R)=T(R, R)$.

Now we provide some useful properties which are equivalent to the IMIP ring property, via the structure of $D_{3}(R)$.

Proposition 2.2. For a ring $R$, the following conditions are equivalent:

(1) $R$ is a reduced ring;

(2) $D_{3}(R)$ is Armendariz;

(3) $D_{3}(R)$ is IFP;

(4) $D_{3}(R)$ is IMIP.

Proof. The equivalence of the conditions (1), (2) and (3) are proved by [11, Proposition 2.8$]$ and $(3) \Rightarrow(4)$ is obvious.

$(4) \Rightarrow(1)$ : Let $D_{3}(R)$ be IMIP and assume on the contrary that there is a nonzero $a \in R$ with $a^{2}=0$. We apply the computation in [11, Proposition 2.8]. Take

$$
A=\left(\begin{array}{ccc}
a & a & -1 \\
0 & a & -1 \\
0 & 0 & a
\end{array}\right), B=\left(\begin{array}{ccc}
a & 0 & a \\
0 & a & 1 \\
0 & 0 & a
\end{array}\right) \in D_{3}(R)
$$

Then $A B=0$ but every maximal ideal of $D_{3}(R)$ contains a nonzero matrix

$$
\left(\begin{array}{ccc}
0 & 1-a & 0 \\
0 & 0 & 0 \\
0 & 0 & 0
\end{array}\right)
$$

So we obtain

$$
\left(\begin{array}{ccc}
a & a & -1 \\
0 & a & -1 \\
0 & 0 & a
\end{array}\right)\left(\begin{array}{ccc}
0 & 1-a & 0 \\
0 & 0 & 0 \\
0 & 0 & 0
\end{array}\right)\left(\begin{array}{ccc}
a & 0 & a \\
0 & a & 1 \\
0 & 0 & a
\end{array}\right)=\left(\begin{array}{ccc}
0 & 0 & a \\
0 & 0 & 0 \\
0 & 0 & 0
\end{array}\right) \neq 0
$$

This induces a contradiction to $D_{3}(R)$ being IMIP. Thus $R$ is reduced.

Based on Proposition 2.2, we may ask whether $D_{n}(R)$ is also IMIP for $n \geq 4$ when $R$ is a reduced ring. However the following erases the possibility. 
Proposition 2.3. For a ring $R$, we have the following results.

(1) $D_{n}(R)$ is not IMIP for any ring $R$ when $n \geq 4$.

(2) If $D_{2}(R)$ is IMIP, then $R$ is IFP.

Proof. (1) Let $R$ be any ring and $n \geq 4$. Then every maximal ideal of $D_{n}(R)$ must contains $E_{23}$. So we have $E_{12} E_{23} E_{34} \neq 0$. But $E_{12} E_{34}=0$ in $D_{n}(R)$, hence $D_{n}(R)$ is not IMIP for $n \geq 4$.

(2) Note that any maximal ideal of $D_{2}(R)$ is of the form

$$
\left\{\left(\begin{array}{ll}
a & r \\
0 & a
\end{array}\right) \mid a \in M \text { and } r \in R\right\} \text {, }
$$

where $M$ denotes a maximal ideal of $R$. Suppose that $D_{2}(R)$ is IMIP and let $a b=0$ for $a, b \in R$. Then

$$
\left(\begin{array}{ll}
a & 0 \\
0 & a
\end{array}\right)\left(\begin{array}{ll}
b & 0 \\
0 & b
\end{array}\right)=0
$$

in $D_{2}(R)$. We use $X$ to denote $\left\{\left(\begin{array}{cc}m & r \\ 0 & m\end{array}\right) \mid m \in M, r \in R\right\}$. Since $D_{2}(R)$ is IMIP, for any maximal ideal $M$ of $R$ we have

$$
\left(\begin{array}{ll}
a & 0 \\
0 & a
\end{array}\right) X\left(\begin{array}{ll}
b & 0 \\
0 & b
\end{array}\right)=0
$$

This implies $a R b=0$, showing that $R$ is IFP.

The condition " $R$ is IFP" in Proposition $2.3(2)$ cannot be replaced by the condition " $R$ is reduced" by the following example.

Example 2.4. Let $R=\mathbb{Z}_{4}$. Then $R$ is not reduced (but IFP) with the only maximal $M=\{0,2\}$. Take any two nonzero elements

$$
A=\left(\begin{array}{ll}
a & b \\
0 & a
\end{array}\right) \text { and } B=\left(\begin{array}{ll}
c & d \\
0 & c
\end{array}\right) \in D_{2}(R)
$$

with $A B=0$. Then $A B$ is one of the following

$$
\left(\begin{array}{ll}
0 & 2 \\
0 & 0
\end{array}\right)\left(\begin{array}{ll}
2 & d \\
0 & 2
\end{array}\right),\left(\begin{array}{ll}
2 & b \\
0 & 2
\end{array}\right)\left(\begin{array}{ll}
0 & 2 \\
0 & 0
\end{array}\right),\left(\begin{array}{ll}
2 & 0 \\
0 & 2
\end{array}\right)\left(\begin{array}{ll}
2 & 0 \\
0 & 2
\end{array}\right) \text { or }\left(\begin{array}{ll}
2 & 2 \\
0 & 2
\end{array}\right)\left(\begin{array}{ll}
2 & 2 \\
0 & 2
\end{array}\right)
$$

for any $b, d \in R$. We use $X$ to denote $\left\{\left(\begin{array}{cc}m & r \\ 0 & m\end{array}\right) \mid m \in M, r \in R\right\}$. For the only maximal ideal $X$ of $D_{2}(R)$, we have $A X B=0$, since $4 M=0$. This shows that $D_{2}(R)$ is IMIP but $R$ is not reduced.

The converse of Proposition 2.3(2) need not hold by next example.

Example 2.5. We adopt the ring $R$ and the argument in [9, Example 2]. Let

$$
A=\mathbb{Z}_{2}\left\langle a_{0}, a_{1}, a_{2}, b_{0}, b_{1}, b_{2}, c\right\rangle
$$

be the free algebra generated by noncommuting indeterminates $a_{0}, a_{1}, a_{2}, b_{0}$, $b_{1}, b_{2}, c$ over $\mathbb{Z}_{2}$. Next let $I$ be the ideal of $A$ generated by

$$
\begin{aligned}
& a_{0} b_{0}, a_{0} b_{1}+a_{1} b_{0}, a_{0} b_{2}+a_{1} b_{1}+a_{2} b_{0}, a_{1} b_{2}+a_{2} b_{1}, a_{2} b_{2}, a_{0} r b_{0}, a_{2} r b_{2}, \\
& b_{0} a_{0}, b_{0} a_{1}+b_{1} a_{0}, b_{0} a_{2}+b_{1} a_{1}+b_{2} a_{0}, b_{1} a_{2}+b_{2} a_{1}, b_{2} a_{2}, b_{0} r a_{0}, b_{2} r a_{2},
\end{aligned}
$$




$$
\left(a_{0}+a_{1}+a_{2}\right) r\left(b_{0}+b_{1}+b_{2}\right),\left(b_{0}+b_{1}+b_{2}\right) r\left(a_{0}+a_{1}+a_{2}\right), \text { and } r_{1} r_{2} r_{3} r_{4},
$$

where the constant terms of $r, r_{1}, r_{2}, r_{3}, r_{4} \in A$ are zero. Now set $R=A / I$. Then $R$ is an IFP ring by the argument in [9, Example 2].

We identity $a_{0}, a_{1}, a_{2}, b_{0}, b_{1}, b_{2}, c$ with their images in $R$ for simplicity. Consider the extension ring $D_{2}(R)$ of $R$. Let

$$
A=\left(\begin{array}{cc}
a_{0} & a_{1} \\
0 & a_{0}
\end{array}\right) \text { and } B=\left(\begin{array}{cc}
b_{0} & b_{1} \\
0 & b_{0}
\end{array}\right) \in D_{2}(R) .
$$

Then $A B=0$, but every maximal ideal of $D_{2}(R)$ contains $\left(\begin{array}{cc}r & 0 \\ 0 & r\end{array}\right)$ where $0 \neq r \in$ $R$ and so

$$
A\left(\begin{array}{cc}
a_{1} & 0 \\
0 & a_{1}
\end{array}\right) B=\left(\begin{array}{cc}
0 & a_{0} a_{1} b_{1}+a_{1} a_{1} b_{0} \\
0 & 0
\end{array}\right) \neq 0
$$

since $a_{0} a_{1} b_{1}+a_{1} a_{1} b_{0} \neq 0$ by the construction of $I$. This implies that $D_{2}(R)$ is not IMIP.

The following example shows that the class of IMIP rings is not closed under homomorphic images.

Example 2.6. Consider the ring $R=D_{3}(\mathbb{Z})$. Then $R$ is IMIP by Proposition 2.3 since $\mathbb{Z}$ is reduced. Let $I=D_{3}\left(2^{2} \mathbb{Z}\right)$. Then $I$ is an ideal of $R$ such that $R / I \cong D_{3}\left(\mathbb{Z}_{4}\right)$. But $D_{3}\left(\mathbb{Z}_{4}\right)$ cannot be IMIP by Proposition 2.3 because $\mathbb{Z}_{4}$ is not reduced.

It is natural to conjecture that $R$ is an IMIP ring if for any nonzero proper ideal $I$ of $R, R / I$ and $I$ are IMIP, where $I$ is considered as an IMIP ring without identity. However we have a negative answer to this question by the next example.

Example 2.7. Consider the non-IMIP ring $R=U_{2}(D)$ over a division ring $D$, as in Example 1.8. The only nonzero proper ideals of $R$ are

$$
I_{1}=\left(\begin{array}{cc}
D & D \\
0 & 0
\end{array}\right), I_{2}=\left(\begin{array}{cc}
0 & D \\
0 & D
\end{array}\right) \text { and } I_{3}=\left(\begin{array}{cc}
0 & D \\
0 & 0
\end{array}\right),
$$

and they are IFP by $\left[9\right.$, Example 5] and so IMIP. Notice that $R / I_{1}$ and $R / I_{2}$ are isomorphic to $D$ and $R / I_{3}=\left\{\left(\begin{array}{cc}a & 0 \\ 0 & c\end{array}\right)+I_{3} \mid a, c \in D\right\}$ is a reduced ring. Therefore each $R / I_{i}$ (for $i=1,2,3$ ) is IMIP.

Proposition 2.8. If a ring $R$ has a maximal ideal which is reduced as a subring of $R$, then $R$ is IMIP.

Proof. We apply the proof of [9, Theorem 6]. Assume that $M$ is a maximal ideal of $R$ which is reduced as a subring of $R$. Let $a b=0$ for $a, b \in R$. Then $(b M a)^{2}=0$ and $b M a \subseteq M$. This yields $b M a=0$ since $M$ is reduced. Accordingly, $((a M b) M)^{2}=a M(b M a) M b M=0$ and so $a M b M=0$ since $M$ is reduced. This yields $(a M b)^{2} \subseteq a M b M=0$. But $a M b \subseteq M$, so we get $a M b=0$ since $M$ is reduced. Thus $R$ is IMIP. 
The condition "a maximal ideal which is reduced" in Proposition 2.8 cannot be dropped by Example 2.7. In fact, $I_{1}$ and $I_{2}$ are all maximal ideals but not reduced clearly.

It is natural to ask whether the class of IMIP rings is closed direct sums. But the answer is negative by next example.

Example 2.9. We take the IMIP ring $R=\operatorname{Mat}_{2}\left(\mathbb{Z}_{2}\right)$ as in Example 2.1. Consider $R \oplus R$. The only maximal ideals are $R \oplus\{0\}$ and $\{0\} \oplus R$. Let

$$
A=\left(\begin{array}{ll}
1 & 1 \\
1 & 1
\end{array}\right) \in R \oplus R
$$

Note that $A^{2}=0$. Then $(A, A)(A, A)=0$ in $R \oplus R$. For

$$
\alpha=\left(\begin{array}{ll}
1 & 0 \\
0 & 0
\end{array}\right) \in R
$$

$(\alpha, 0) \in R \oplus\{0\}$, and $(0, \alpha) \in\{0\} \oplus R$. Then $(A, A)(\alpha, 0)(A, A)=(A, 0)$ and $(A, A)(0, \alpha)(A, A)=(0, A)$, showing that $(A, A)(R \oplus\{0\})(A, A) \neq 0$ and $(A, A)(\{0\} \oplus R)(A, A) \neq 0$, respectively. Thus $R \oplus R$ is not IMIP.

By the similar argument to above, it can be shown that a direct sum $R=$ $\bigoplus_{\gamma \in \Gamma} R_{\gamma}$ of IMIP rings $R_{\gamma}$ for an indexed set $\Gamma$ is not IMIP.

Proposition 2.10. If $R[x]$ over a ring $R$ is an IMIP ring, then so is $R$.

Proof. Suppose that $R[x]$ is IMIP and let $M$ be a maximal ideal of $R[x]$. Set

$$
M_{0}=\{a \in R \mid a \text { is the constant term of } f(x) \text { when } f(x) \in M\} .
$$

Since $M$ is a maximal ideal of $R[x]$, we have either $M_{0}$ is a maximal ideal of $R$ or $M_{0}=R$. Now, let $a b=0$ for $a, b \in R$. By assumption, $a M b=0$ and so either $a M_{0} b=0$ or $a R b=0$. Each case of them entails that $R$ is IMIP.

But, the following example illuminates that the IMIP ring property does not go up to polynomial rings and power series rings.

Example 2.11. (1) Recall the IFP ring $R$ in Example 2.5 and apply the argument in [9, Example 2]. Let $M$ be the set of all polynomials in $R$ with zero constant term. Then $M$ is the unique maximal ideal of $R$ since $M^{4}=0$ and any $r \in R \backslash M$ is a unit. Note $R / M \cong \mathbb{Z}_{2}$.

If we take

$$
f(x)=a_{0}+a_{1} x+a_{2} x^{2} \text { and } g(x)=b_{0}+b_{1} x+b_{2} x^{2}
$$

in $R[x]$, then $f(x) g(x)=0$ as we see in the computation in Example 2.5. Since $M[x]$ is an ideal of $R[x]$ with $M[x]^{4}=0$, every maximal ideal $P$ of $R[x]$ contains $M[x]$, especially $c$. Recall $f(x) c g(x) \neq 0$. This implies $f(x) P g(x) \neq 0$, showing that $R[x]$ is not IMIP.

(2) Consider the power series ring $R[[x]]$ over an IMIP ring $R(1)$. Observe that every maximal ideal $N$ of $R[[x]]$ is of the form $M+x R[[x]]$ with a maximal ideal $M$ of $R$. By (1), there exist $f(x), g(x) \in R[[x]]$ such that $f(x) g(x)=0$ 
and $f(x) c g(x) \neq 0$. Then $0 \neq f(x) \operatorname{cxg}(x) \in f(x) N g(x)$, concluding that $R[[x]]$ is not IMIP.

It is well-known that the IFP ring which is Armendariz can go up to polynomials. We do not know an example of an Armendariz IMIP ring $R$ such that $R[x]$ is not IMIP.

Question. If $R$ is IMIP and Armendariz, then is $R[x]$ IMIP?

The concepts of an Armendariz ring and an IMIP ring do not imply each other by the following.

Example 2.12. (1) For a simple ring $A, R=\operatorname{Mat}_{n}(A)(n \geq 2)$ is IMIP by Theorem 1.6, but $R$ is not Armendariz by [4, Remark 3.1] (or, see [9, Example $2])$.

(2) The $\operatorname{ring} R=U_{3}(A)$ over a reduced $\operatorname{ring} A$ is Armendariz by [13, Proposition 2], but not IMIP by Example 1.8.

Recall that the Abelian ring is also a generalization of IFP rings. But the concepts of IMIP and Abelian are independent on each other by the following example and Remark 1.7(1).

Example 2.13. We use the subring

$$
R=\left\{\left(\begin{array}{ll}
a & c \\
0 & b
\end{array}\right) \mid a-b \equiv c \equiv 0(\bmod 2)\right\}
$$

of $\operatorname{Mat}_{2}(\mathbb{Z})$ in $[13$, Example 13]. Then $R$ is Abelian by the argument in [13, Example 13]. Let $M$ be any maximal ideal of $R$. Then $M$ must contain a matrix

$$
\left(\begin{array}{ll}
\alpha & \beta \\
0 & \gamma
\end{array}\right) \text { with } \beta \neq 0
$$

So we have

$$
\left(\begin{array}{ll}
2 & 0 \\
0 & 0
\end{array}\right)\left(\begin{array}{ll}
0 & \beta \\
0 & 0
\end{array}\right)\left(\begin{array}{ll}
0 & 0 \\
0 & 2
\end{array}\right)=\left(\begin{array}{cc}
0 & 4 \beta \\
0 & 0
\end{array}\right) \neq 0 .
$$

This entails

$$
\left(\begin{array}{ll}
2 & 0 \\
0 & 0
\end{array}\right) M\left(\begin{array}{ll}
0 & 0 \\
0 & 2
\end{array}\right) \neq 0, \text { but }\left(\begin{array}{ll}
2 & 0 \\
0 & 0
\end{array}\right)\left(\begin{array}{ll}
0 & 0 \\
0 & 2
\end{array}\right)=0
$$

Thus $R$ is not IMIP.

Acknowledgments. The authors thank the referee for very careful reading of the manuscript and many valuable suggestions that improved the paper by much. The first named author was supported by the National Natural Science Foundation of China(11361063). And the third named author was supported by Basic Science Research Program through the National Research Foundation of Korea(NRF) funded by the Ministry of Education(NRF2013R1A1A2A10004687). 


\section{References}

[1] D. D. Anderson and V. Camillo, Armendariz rings and Gaussian rings, Comm. Algebra 26 (1998), no. 7, 2265-2272.

[2] E. P. Armendariz, A note on extensions of Baer and P.P.-rings, J. Austral. Math. Soc. 18 (1974), 470-473.

[3] H. E. Bell, Near-rings in which each element is a power of itself, Bull. Austral. Math. Soc. 2 (1970), 363-368.

[4] S. Chhawchharia and M. B. Rege, Armendariz rings, Proc. Japan Acad. Ser. A Math. Sci. 73 (1997), no. 1, 14-17.

[5] J. L. Dorroh, Concerning adjunctins to algebras, Bull. Amer. Math. Soc. 38 (1932), $85-88$.

[6] K. R. Goodearl, Von Neumann Regular Rings, Pitman, London, 1979.

[7] J. Hannah and K. C. O'meara, Maximal quotient rings of prime group algebras, Proc. Amer. Math. Soc. 65 (1977), no. 1, 1-7.

[8] C. Huh, S. H. Jang, C. O. Kim, and Y. Lee, Rings whose maximal one-sided ideals are two sided, Bull. Korean Math. Soc. 39 (2002), no. 3, 411-422.

[9] C. Huh, Y. Lee, and A. Smoktunowicz, Armendariz rings and semicommutative rings, Comm. Algebra 30 (2002), no. 2, 751-761.

[10] S. U. Hwang, Y. C. Jeon, and Y. Lee, Structure and topological conditions of NI rings, J. Algebra 302 (2006), no. 1, 186-199.

[11] Y. C. Jeon, H. K. Kim, Y. Lee, and J. S. Yoon, On weak Armendariz rings, Bull. Korean Math. Soc. 46 (2009), no. 1, 135-146.

[12] M. Kheradmand, H. K. Kim, T. K. Kwak, and Y. Lee, Reflexive property on nil ideals, Submitted.

[13] N. K. Kim and Y. Lee, Armendariz rings and reduced rings, J. Algebra 223 (2000), no. $2,477-488$.

[14] Extensions of reversible rings, J. Pure Appl. Algebra 185 (2003), no. 1-3, 207223.

[15] L. Motais de Narbonne, Anneaux semi-commutatifs et unis riels anneaux dont les id aux principaux sont idempotents, Proceedings of the 106th National Congress of Learned Societies (Perpignan, 1981), 71-73, Bib. Nat., Paris, 1982.

[16] G. Shin, Prime ideals and sheaf representation of a pseudo symmetric ring, Trans. Amer. Math. Soc. 184 (1973), 43-60.

[17] A. Smoktunowicz, A simple nil ring exists, Comm. Algebra 30 (2002), no. 1, 27-59.

HAI-LAN JIN

Department of Mathematics

YANBIAN UNIVERSITY

YANJI 133002, P. R. CHINA

E-mail address: hljin98@hanmail.net

DA WOON JunG

Department of Mathematics

Pusan National University

Pusan 609-735, Korea

E-mail address: jungdw@pusan.ac.kr 
YANG LEE

Department of Mathematics

Pusan National University

PUSAN 609-735, KorEA

E-mail address: ylee@pusan.ac.kr

Sung Ju Ryu

Department of Mathematics

Pusan National University

PusAn 609-735, Korea

E-mail address: sjryu@pusan.ac.kr

HyO JiN SUNG

Department of Mathematics

Pusan National University

Pusan 609-735, Korea

E-mail address: hjsung@pusan.ac.kr

SANG JO YUN

Department of Mathematics

Pusan National University

Pusan 609-735, Korea

E-mail address: pitt0202@hanmail.net 